\title{
LAS REFORMAS NEOGERENCIALES EN HISPANOAMERICA. ENSEÑANZAS PARA INCREMENTAR LAS CAPACIDADES DE GOBIERNO
}

\section{José Juan Sánchez González}

Universidad Autónoma del Estado de México

\section{Resumen}

El artículo analiza, críticamente, los procesos de reformas impulsados, bajo la lógica de la Nueva Gestión Pública, en el mundo occidental y en Hispanoamérica. Se argumenta que el modelo neogerencial fue percibido como universal, y replicable a cualquier país, sin importar el nivel de desarrollo administrativo, el grado de profesionalización de la burocracia, además que no consideraba el nivel de democracia y de participación ciudadana en los asuntos del gobierno. El modelo estaba orientado más al cliente y la incorporación de la competencia en el mercado de los bienes. Sin embargo, descuidó lo fundamental de toda acción de gobierno: consolidar las capacidades institucionales para coadyuvar en la democracia. Ello no significa descartar los procesos desarrollados. Debido a que algunos fueron exitosos, es necesario destacar las enseñanzas y aprendizajes que estas experiencias pueden entregar, para mejorar la capacidad de gobierno en sociedades democráticas.

Palabras Claves: Administración Pública, Nueva Gestión Pública, Reformas Neogerenciales, Hispanoamérica.

\section{Abstract}

The paper analyzes, from a critical perspective, reform processes based on New Public Management ideas, promoted in the Western world and Hispanic America. It is argued that the neomanagerial model was perceived as universal and replicable in any country, without considering the levels of administrative development, bureaucracy professionalization, democracy or citizenry participation in government affairs. The model was more focused on the client and the incorporation of competition within goods markets. However, it overlooked the main feature of any government action: to consolidate institutional capabilities to support democracy. This does not imply ruling out the processes developed so far. Given that some of them were successful, it is necessary to highlight the lessons and learning that these experiences can bring us, in order to improve government capabilities in democratic societies.

Keywords: Public Administration, New Public Management, Neomanagerial Reforms, Spanish 
America.

\section{Presentación}

El modelo neogerencial, basado en la Nueva Gestión Pública (NGP), fue percibido como universal, y replicable a cualquier país sin importar el nivel de desarrollo administrativo, centralizado o descentralizado, sin tomar en cuenta el grado de profesionalización de la burocracia, además que no consideraba el nivel de democracia y de participación ciudadana en los asuntos del gobierno. El modelo estaba orientado más al cliente y la incorporación de la competencia en el mercado de los bienes y servicios en el mercado, la concesión de servicios públicos que pudieran ser realizados por particulares, así como la medición del desempeño y de los resultados, entre otros. Sin embargo, descuidó lo fundamental de toda acción de gobierno: consolidar las capacidades institucionales para coadyuvar en la democracia.

El tránsito desde el modelo "oficina" al modelo neogerencial en la administración pública de Hispanoamérica tiene enseñanzas y aprendizajes en el contexto de las reformas administrativas para mejorar la capacidad de gobierno en sociedades democráticas. En ese sentido, sostenemos la tesis de que en la medida que algunas de estas reformas neogerenciales tuvieron éxito, en esa misma proporción pueden contribuir a mejorar la capacidad de gobierno y fortalecer la democracia en Hispanoamérica. La modernización de la administración pública como un aparato eficiente, eficaz, oportuno, orientado al ciudadano, con rendición de cuentas y transparencia en la información, repercute de manera proactiva en la consolidación de las instituciones democráticas.

La estructura del ensayo para abordar este tema es el siguiente: primero, revisamos de manera sucinta el desarrollo teórico desde la Gestión Pública a la NGP, destacando algunos de los autores más representativos; segundo, se revisan las reformas gubernamentales en el mundo a partir de cuatro modelos, tanto de países desarrollados como de reciente industrialización; tercero, se describen las reformas neogerenciales efectuadas en países como Argentina, Chile, Bolivia, Colombia, Nicaragua, México, Uruguay y Venezuela; cuarto, tratamos de responder a la pregunta de si las reformas neogerenciales contribuyeron a mejorar las capacidades del gobierno; por último, sugerimos algunas ideas que se consideran como conclusiones.

\section{La Nueva Gerencia Pública: una oleada de mercados, clientes y eficacia en la administración pública}

El término NGP expresa la idea del caudal acumulado de decisiones sobre políticas a seguir en los pasados veinte años, y que ha redundado en un giro sustancial en el ejercicio del gobierno 
y la decisión del sector estatal en el Reino Unido, Nueva Zelanda, Australia, Escandinavia y América del Norte. La NGP es una fórmula sintética, utilizada regularmente por especialistas y profesionales, para referirse a los temas, estilos y patrones distintivos de la gestión de la función pública que tomaron delantera durante las dos últimas décadas, especialmente en países anglosajones (Barzelay, 2001: 16), y es la más moderna de las vertientes administrativas del modelo económico vigente en muchos países del mundo (Martínez, 2007: 33). EI NGP es nuevamente un gran foco de interés teórico y práctico. Aunque su estudio es cultivado en países anglófonos -Estados Unidos, Reino Unido, Canadá y Australia-, su influencia abraza otras naciones (Guerrero, 1999: 89).

Las explicaciones sobre el desarrollo de la administración pública recibieron una sacudida cuando muchos países de la OCDE empezaron a moverse hacia lo que llegó a conocerse como la NGP. La terminología se acuñó para etiquetar un cambio en los estilos de gestión pública. Se intentaba que fuera un término común en el lenguaje particular con el que los consultores definían las reformas del sector público en cada país (como el Projet de Service, de Francia, el Next Steps, de Gran Bretaña, o el Public Service 2000, de Canadá) y subrayar sus rasgos compartidos. Se pueden establecer analogías con los términos "nueva política" o "nueva derecha" y "nuevo Estado industrial", que fueron inventados por razones similares (Hood, 1994; 473).

El desarrollo teórico desde la Gerencia Pública a la NGP ha sido producto de un conjunto de autores y obras que han aportado conocimiento para mejorar la administración pública contemporánea (Sánchez, 2006). Analizando los diferentes paradigmas -tradicional, eficientista y de calidad- propuestos por Richards (1994) y retomados por Haywood y Rodríguez (1994), se puede identificar el proceso evolutivo, desde la gerencia pública a la NGP.

Los cambios en la administración pública se iniciaron, cada vez más fuertes, desde la comunidad internacional, quien empezó a considerar prioritaria la reforma neogerencial, entre ellos: la Organización para la Cooperación y el Desarrollo Económico (OCDE); el Banco Mundial, el Programa de Naciones Unidas para el Desarrollo, el Banco Interamericano de Desarrollo, la Unión Europea y muchos órganos regionales, así como los donadores de asistencia bilateral, los socios comerciales y las ONG (Lynn, 2004: 106). La NGP se gestó cronológicamente a finales del siglo XX, aunque la revolución gerencial es un proceso propio del siglo XXI (Guerrero, 2003a; 20).

Desde el punto de vista teórico, a partir de la propuesta de Barzelay (2001) se pueden establecer diversos momentos relevantes en el campo de la NGP. En primer lugar, los dos primeros trabajos -Hood y Jackson (1997) y el de Aucoin (1990)- como pioneros en la conformación de este nuevo paradigma. En segundo lugar, aparecen las obras de Osborne y Gaebler (1992) y Barzelay (1992), como documentos difusores de las nuevas ideas de la NGP, 
los cuales describen el caso de Estados Unidos. En tercer lugar, se agrupan las investigaciones que tienen que ver con estudios de casos en países anglosajones, Nueva Zelanda (1991), Australia (1992), el análisis comparativo de la NGP (1994), realizado por Schwartz donde revisa las similitudes entre Australia, Nueva Zelanda, Suecia y Dinamarca, en los años 1980, posteriormente, los casos de Reino Unido (1995), Canadá (1995) y Estados Unidos (1995), así como dos casos fuera de la esfera anglosajona: México (2000) y Brasil (1994). En cuarto lugar, destacan la importancia de los trabajos críticos de Bozeman (1993), Hood (1994), Moore (1998) y Lynn (1996), que sirven como marco de referencia para posteriores polémicas en torno al tema. Por último, Barzelay (2001) incluye algunos textos publicados en el año de 1996 como el de Ferlie (1996) y Schick (1996), así como los de Bardach (1998), Pollitt y Bouckaert (2000), y el propio Barzelay (2000).

En tanto andamiaje teórico, la NGP puede ser interpretada a partir del perfil siguiente: 1) es una visión privada de lo público, de naturaleza cataláctica y que proviene del pensamiento económico clásico, que la sitúa en el mercado para sustituir el esquema burocrático; 2) se ostenta como nueva pero sus antecedentes son tan antiguos como la economía clásica y neoclásica; 3 ) en ella convergen una variedad de ingredientes pero de igual naturaleza, lo que la hace compleja; 4) su arribo es consonante con los tiempos actuales cuando se proponen soluciones económicas a los problemas políticos en una atmósfera donde el estudio de la política se distancia de la economía; 5) son conceptos fundamentales la orientación al cliente, privatización, mercado y competencia, reforma del sector público, enfoque empresarial-gerencial, gerencia por objetivos y resultados, y agenciación y reducción de costos; 6) y situada en el mercado, paradójicamente desde dentro es activada por la gerencia y da paso a reglas burocráticas, reemplazando al gobierno de hombres por un gobierno sin política (Guerrero, 2003b: 379).

Una manera de diferenciar a la administración pública y la nueva gerencia pública es mediante el análisis comparativo de algunos criterios que se muestran en la siguiente Tabla:

TABLA 1: Diversidades entre la administración pública y la Nueva Gerencia Pública

\begin{tabular}{|l|l|l|}
\hline Carácter distintivo & Administración Pública & Nueva Gerencia Pública \\
\hline Principio causal & La política & La economía \\
\hline Foco organizativo & $\begin{array}{l}\text { El Estado: relaciones de } \\
\text { dominación }\end{array}$ & $\begin{array}{l}\text { El mercado: relaciones de } \\
\text { intercambio }\end{array}$ \\
\hline Núcleo procesal & La jerarquía: cadena de delegación & La catalaxia: contrato \\
\hline Corazón activo & Servicios públicos & Competencia \\
\hline Fundamento jurídico & Derecho administrativo & Derecho mercantil \\
\hline Orientación hacia el ser humano & Lo público & Lo privado \\
\hline Fundamento axiológico & La ética: bienestar público & La eficiencia: individualismo \\
\hline Naturaleza científica & Ciencia social & Transdisciplina económica \\
\hline
\end{tabular}

Fuente: Guerrero (2003b: 390). 
La NGP consiste en un modelo constituido de manera opuesta a la administración pública -como lo muestra la Tabla 1- ya que ésta tiene como principio causal la política, su foco organizativo es el Estado, con sus relaciones de dominación; y su núcleo procesal es la jerarquía, es decir, una cadena de delegación; mientras que en la NGP, la economía es la unidad de objeto, el mercado con sus relaciones de intercambio es el foco organizativo, y la catalaxia es el núcleo procesal con sus relaciones contractuales.

El corazón activo de la administración pública es el servicio público, el de la gerencia es el mercado; la primera, tiene una vocación regulatoria y está normada por el derecho administrativo; la segunda, está inspirada en la desregulación y su escenario formal es el derecho mercantil. La administración pública detenta sus valores en la ética y produce un efecto social de bienestar colectivo, en tanto que la NGP exalta la eficacia y su efecto económico se orienta al elogio del individualismo; "La primera es pública y colectiva, la segunda privada y egoísta. La una está basada en una disciplina dentro de las ciencias sociales, la otra es una transdisciplina económica que ha colonizado a la administración pública" (Guerrero, 2003b: 390).

\section{Reformas gubernamentales en el mundo}

La mayoría de los procesos de reforma gubernamental que se han llevado a cabo en gran parte de los países del mundo a lo largo de las últimas tres décadas, están inspiradas en las ideas, técnicas y metodologías, postuladas por la NGP (Cabrero, 2005: 1). Esta ola de reformas gubernamentales ha dominado el escenario internacional, a partir de la convicción de que gobiernos que sean evaluados por resultados, que funcionen en situaciones de cuasimercado, que sean ágiles en su accionar, que adopten algunas de las técnicas administrativas que el sector privado ha diseñado, y que se enfoquen al cliente, lograrán mayor eficiencia en su funcionamiento, mejores resultados en los objetivos buscados, así como mayor legitimidad y aceptación por parte de la ciudadanía.

Algunas experiencias exitosas y la insistencia de organismos internacionales en la adopción de este tipo de estrategias neogerenciales, han contribuido a un "efecto imitación" o de isomorfismo, el cual ha llegado a una gran diversidad de países. Tanto los gobiernos de países desarrollados como emergentes, aspiran y se esfuerzan por adoptar "recetas" como un posible camino más rápido y eficaz para construir gobiernos que "cuesten menos y hagan más".

Las reformas gubernamentales en el mundo han tenido diversos enfoques, aunque los estudios de caso como Nueva Zelanda, Australia, Reino Unido, Estados Unidos y Canadá, constituyen el núcleo de experiencias en NGP. Tomando estas referencias y de otros países, Conde (2001) establece cuatro modelos: Anglosajón, Europeo Continental, Europeo Septentrional y Países en 
Transición, que permiten mostrar la diversidad de experiencias y establecer la hipótesis de que la NGP no es un modelo universal y aplicable a todos los países (Conde, 2001: 373).

El primer modelo es el Anglosajón, en el cual los países de habla inglesa comparten una estrategia radical de reforma de la gestión pública basada en la doctrina de la NGP. Desde el punto de vista teórico, se asumen los esquemas de la teoría de la elección pública que propugna la desburocratización de los aparatos públicos y la recuperación del control de las organizaciones por los responsables políticos (Conde, 2001: 375). Por lo que se refiere a la práctica administrativa, se parte de los postulados de los estudios de management en su versión norteamericana, que sostiene la superioridad de la gestión privada sobre la pública. Los casos representativos son los de Nueva Zelanda, Australia, Gran Bretaña e Irlanda.

El segundo modelo es el Europeo Continental, que puede ser definido como de reforma incremental. El modelo clásico de administración pública se ve modificado por determinados cambios graduales y parciales que no afectan a los elementos constitutivos del mismo. En los países de tradición napoleónica, ciertos rasgos estructurales continúan vigentes: gestión administrativa, seguridad en el empleo y servicio civil de carrera. Dentro de este contexto, la gerencia pública y la gerencia privada se consideran fundamentalmente diferentes. Existe una diferencia en la percepción del papel del Estado (Conde, 2001; 380). Mientras que en la perspectiva liberal que inspira la NGP del mundo anglosajón da lugar a una reducción del papel del Estado en la economía y una retirada del ámbito económico y social, las reformas del continente europeo van dirigidas a reforzar la legitimidad de la intervención del Estado, a través de formas de acción renovadas.

Por ejemplo, Grecia e Italia dirigen sus esfuerzos a reforzar las estructuras estatales dentro de su lucha contra la corrupción y por el saneamiento de la vida pública. Hasta cierto punto, en su caso se trata de reforzar la administración weberiana y el control de legalidad de las administraciones. Por el contrario, en otros países, como Francia, Alemania, España o Portugal el reto es más bien desburocratizar y agilizar unas administraciones cuya orientación no se dirige con suficiente intensidad al logro de objetivos y a la obtención de un rendimiento satisfactorio.

El tercer modelo es el Europeo Septentrional. En este grupo se encuentran los países escandinavos y los Países Bajos. Estos optan por un modelo próximo a la NGP, pero dotándolo de un perfil pragmático que la separa de la concepción radical del ámbito anglosajón (Conde, 2001; 384). Este conjunto de países no rechaza de modo categórico el modelo de gestión pública tradicional, sino que cuestiona su utilidad en algunos ámbitos de la acción administrativa, sustituyéndolo por otras fórmulas basadas en la eficiencia.

En el caso de los Países Bajos existe una voluntad de reforma que se mantiene a lo largo de las décadas de 1980 y 1990. El objetivo global es la reducción del tamaño y alcance del gobierno 
a través de políticas de privatización, la creación de agencias y el análisis de las diferentes formas de intervención y gasto. En 1982, fue lanzada la iniciativa Grandes Operaciones, dirigida al logro de seis objetivos: 1) reconsideración del gasto público; 2) reorganización de los servicios administrativos centrales; 3) desregulación de la intervención gubernamental; 4) descentralización; 5) desburocratización de las organizaciones estatales; y 6) inicio de un proceso de privatizaciones.

El cuarto modelo es el de un grupo, Países en Transición, con diversos niveles de desarrollo que están realizando transformaciones institucionales, tanto en el campo político como en el económico (Conde, 2001: 386-387). Por una parte, se encuentran México y Turquía, países que, con ciertas salvedades, muestran significativas similitudes. En primer lugar, el menor nivel de desarrollo en términos comparativos los enfrenta al reto económico, el cual en ambos casos está fuertemente determinado por su integración en espacios económicos y comerciales abiertos, como es el europeo y estadounidense. En segundo lugar, ambos países asumen principios democráticos cuyo traslado a la práctica cotidiana resulta aún insuficiente en diversos ámbitos, por lo que la profundización democrática forma parte de su agenda política.

Por otra parte, hay que referirse a los ex países comunistas europeos: Polonia, Hungría y República Checa. La transición de estos países, profunda en términos políticos y económicos, afecta cada vez más directamente a los aparatos administrativos del gobierno. Primero, porque se entiende que una administración pública eficiente es una condición indispensable para obtener un desarrollo económico adecuado. Segundo, porque la reforma de las estructuras y la gestión administrativa es una condición impuesta para el ingreso a la Unión Europea.

Por último, hay que mencionar el caso de Corea. Se trata de un país en transición en el que un rápido crecimiento económico protagonizado por un Estado intervencionista ha generado múltiples tensiones políticas y sociales ligadas a las escasas virtudes democráticas del régimen político y a la importancia del problema de la corrupción. La grave crisis económica que sacudió en los últimos años las economías asiáticas ha sido la causa de diversos programas de reforma, durante los años 1990.

Por lo que se refiere a los países latinoamericanos, las reformas de segunda generación de la gestión pública aún no tienen los alcances y pretensiones de los países desarrollados y por supuesto de los realizados en la OCDE. Tal como lo plantea el Centro Latinoamericano de Administración para el Desarrollo (CLAD), los países en desarrollo -particularmente los latinoamericanos-sólo pusieron en marcha en la década de 1980 reformas de primera generación dirigidas principalmente a la reducción del gasto público, redimensionamiento del aparato gubernamental, privatización de empresas públicas y desregulación, para intentar alcanzar una disminución en el déficit público. La crisis fiscal del Estado habría llevado a realizar este tipo de 
reformas, que lograron disminuir los gastos del gobierno pero que tuvieron una repercusión en la capacidad gubernamental, en la oportunidad y calidad de los servicios públicos.

\section{Reformas neogerenciales en Hispanoamérica: un análisis comparativo}

En este apartado, el propósito es revisar las reformas neogerenciales efectuadas en algunos de los países más representativos de la región, como son Argentina, Chile, Bolivia, Colombia, Nicaragua, México, Uruguay y Venezuela (Vicher, 2008: 379). ${ }^{1}$ A partir de esta selección de países, se orienta el análisis para intentar comprender si las mismas reformas contribuyeron a incrementar o disminuir las capacidades de los gobiernos que las impulsaron. Una versión abreviada de las principales estrategias gerenciales, se muestran en la Tabla 2.

TABLA 2: Principales reformas gerenciales en países estudiados

\begin{tabular}{|c|c|c|c|c|c|c|c|c|}
\hline \multirow{2}{*}{ Estrategias } & \multicolumn{8}{|c|}{ Países } \\
\hline & Argentina & Bolivia & Chile & Colombia & Nicaragua & México & Uruguay & Venezuela \\
\hline $\begin{array}{l}\text { Orientación a } \\
\text { resultados }\end{array}$ & $\begin{array}{c}\mathrm{Se} \\
\text { menciona }\end{array}$ & Se aplicó & $\begin{array}{c}\mathrm{Se} \\
\text { menciona }\end{array}$ & $\begin{array}{c}\mathrm{Se} \\
\text { menciona }\end{array}$ & $\begin{array}{c}\mathrm{Se} \\
\text { menciona }\end{array}$ & $\begin{array}{c}\mathrm{Se} \\
\text { menciona }\end{array}$ & $\begin{array}{c}\mathrm{Se} \\
\text { menciona }\end{array}$ & $\begin{array}{c}\mathrm{Se} \\
\text { menciona }\end{array}$ \\
\hline $\begin{array}{l}\text { Simplificación } \\
\text { de estructuras }\end{array}$ & Se aplicó & Se aplicó & $\begin{array}{c}\mathrm{Se} \\
\text { menciona }\end{array}$ & Se aplicó & Se aplicó & Se aplicó & Se aplicó & $\begin{array}{c}\mathrm{Se} \\
\text { menciona }\end{array}$ \\
\hline $\begin{array}{l}\text { Desagregación de } \\
\text { organismos }\end{array}$ & $\begin{array}{c}\mathrm{Se} \\
\text { menciona }\end{array}$ & $\begin{array}{l}\text { No se } \\
\text { aplicó }\end{array}$ & Se aplicó & $\begin{array}{c}\text { No se } \\
\text { menciona }\end{array}$ & $\begin{array}{c}\mathrm{Se} \\
\text { menciona }\end{array}$ & $\begin{array}{c}\mathrm{Se} \\
\text { menciona }\end{array}$ & $\begin{array}{c}\mathrm{Se} \\
\text { menciona }\end{array}$ & Se aplicó \\
\hline Contractualización & Se aplicó & $\begin{array}{c}\text { No se } \\
\text { menciona }\end{array}$ & Se aplicó & Se aplicó & $\begin{array}{c}\text { No se } \\
\text { menciona }\end{array}$ & $\begin{array}{c}\mathrm{Se} \\
\text { menciona }\end{array}$ & $\begin{array}{c}\mathrm{Se} \\
\text { menciona }\end{array}$ & $\begin{array}{c}\mathrm{Se} \\
\text { menciona }\end{array}$ \\
\hline $\begin{array}{l}\text { Descentralización } \\
\text { y flexibilización }\end{array}$ & $\begin{array}{c}\mathrm{Se} \\
\text { menciona }\end{array}$ & Se aplicó & Se aplicó & Se aplicó & $\begin{array}{c}\mathrm{Se} \\
\text { menciona }\end{array}$ & Se aplicó & $\begin{array}{c}\mathrm{Se} \\
\text { menciona }\end{array}$ & Se aplicó \\
\hline $\begin{array}{l}\text { Sistemas de } \\
\text { indicadores y } \\
\text { evaluación }\end{array}$ & Se aplicó & $\begin{array}{c}\mathrm{Se} \\
\text { menciona }\end{array}$ & Se aplicó & Se aplicó & $\begin{array}{c}\mathrm{Se} \\
\text { menciona }\end{array}$ & Se aplicó & Se aplicó & Se aplicó \\
\hline $\begin{array}{l}\text { Rendición de } \\
\text { cuentas }\end{array}$ & $\begin{array}{c}\mathrm{Se} \\
\text { menciona }\end{array}$ & Se aplicó & $\begin{array}{c}\mathrm{Se} \\
\text { menciona }\end{array}$ & $\begin{array}{c}\mathrm{Se} \\
\text { menciona }\end{array}$ & $\begin{array}{c}\text { No Se } \\
\text { menciona }\end{array}$ & $\begin{array}{c}\mathrm{Se} \\
\text { menciona }\end{array}$ & $\begin{array}{c}\mathrm{Se} \\
\text { menciona }\end{array}$ & $\begin{array}{c}\text { No se } \\
\text { menciona }\end{array}$ \\
\hline Mejores prácticas & $\begin{array}{c}\mathrm{Se} \\
\text { menciona }\end{array}$ & $\begin{array}{c}\text { No se } \\
\text { menciona }\end{array}$ & $\begin{array}{c}\mathrm{Se} \\
\text { menciona }\end{array}$ & $\begin{array}{c}\text { No se } \\
\text { menciona }\end{array}$ & $\begin{array}{c}\text { No Se } \\
\text { menciona }\end{array}$ & $\begin{array}{c}\mathrm{Se} \\
\text { menciona }\end{array}$ & $\begin{array}{c}\text { No se } \\
\text { menciona }\end{array}$ & $\begin{array}{c}\text { No se } \\
\text { menciona }\end{array}$ \\
\hline
\end{tabular}

Fuente: Vicher (2008:381)

A partir del análisis excelente del trabajo de investigación de Vicher (2008), se hace una referencia lo más sucinta posible de las reformas realizadas en los siguientes países: Argentina, Chile, Bolivia, Colombia, Nicaragua, México, Uruguay y Venezuela. Para profundizar se sugiere consultar esta obra, que espero muy pronto sea publicada. 
En Argentina, el programa estuvo apoyado y financiado por el Banco Mundial, el Banco Interamericano de Desarrollo (BID), y hombres de negocios, que a través de la Fundación Empresaria para la Modernización del Estado, financiaron la primera fase de la reforma. Además, el programa se consensuó con los economistas más cercanos a los grupos empresariales, quienes avalaron los principales ejes propuestos por el gobierno en esta materia. A su vez, el programa de reforma estuvo gerenciado por un pequeño equipo que contaba con todo el apoyo del Presidente y del Ministro de Economía que, para acelerar la reforma estatal, creó un Comité Ejecutivo Contralor de la Reforma Administrativa (CECRA), presidido por el Ministro de Economía, que era el encargado de dirigir la reforma administrativa.

Cabe señalar que la aplicación de las reformas condujo a grandes inconvenientes en el funcionamiento administrativo, como la superposición de competencias similares en unidades organizacionales diferentes; y agencias burocráticas con límites escasamente delimitados (Vicher, 2008: 250-251). En cuanto al empleo se decía que las reformas fomentarían medidas orientadas a la profesionalización, pero en la práctica la reducción del personal y los recortes presupuestales se impusieron como líneas de acción. Incluso, con la supresión de agencias, en 1991, el número total de Secretarías y Subsecretarías del gobierno argentino se redujo a 65 , pero cinco años después los ocho ministerios existentes reunían en total 189 Secretarías y Subsecretarías, por lo que la evolución de las estructuras administrativas siguió la lógica expansionista.

Por su parte, Chile utilizó las metodologías del Banco Mundial y el BID para diseñar la reforma, y los directivos encargados de implementarla tenían una reconocida trayectoria gerencial en el sector privado, además de que también se contó con el apoyo de consultorías externas. En este país la evolución ha constituido un elemento predominante en lo relativo a la modernización de la gestión pública, lo cual es verificable por la cantidad de iniciativas que se han elaborado. La evolución es una estrategia para el mejoramiento de las instituciones públicas y de la asignación más eficiente y eficaz del gasto público. Sin embargo, y pese al enorme esfuerzo, los avances logrados son escasos y se percibe que la temática de la evaluación aún necesita mayor participación de los gestores públicos (Vicher, 2008: 262).

Por lo que respecta al gobierno de Bolivia, éste fue apoyado y financiado por el Banco Mundial, que colaboró con él para diseñar un programa comprensivo de reforma de gobernanza, es decir, el Proyecto de Reforma Institucional para coadyuvar en la implementación del Plan Nacional de Integridad, que el gobierno anunció en 1998. Con este proyecto se pretende mejorar la efectividad, eficacia y transparencia de la administración pública boliviana, a fin de fortalecer la habilidad del país para implementar programas de desarrollo económico y social que permitan combatir a la pobreza. 
No obstante lo anterior, los resultados de la gestión de las entidades públicas son todavía deficientes y su credibilidad institucional por parte de la ciudadanía es pobre. Se percibe que la administración pública continúa siendo objeto de manipulación por parte de sectores muy reducidos. En este contexto, la corrupción en el aparato gubernamental se manifiesta como uno de los problemas principales que debilitan la confianza ciudadana.

En relación a Colombia, su gobierno retomó preceptos del Banco Mundial, que sustentaba que la NGP contiene las bases necesarias para que el Estado sea eficaz y tenga un desarrollo sostenible en el plano económico y social. El caso de Colombia es un ejemplo clásico de una reforma gerencialista debido a que la NGP continúa siendo un elemento importante dentro de los cambios proyectados para lograr el mejoramiento de la administración pública.

Sin embargo, al año 2003 se señaló que no existía una cultura de gestión de activos que incorpore parámetros de comportamiento empresarial motivados por la renta y la utilización eficiente de los bienes. Incluso, que las entidades públicas preferían incrementar la tendencia de activos, porque, entre otras razones, pueden presionar presupuestos cada vez más altos para el mantenimiento y aseguramiento de los mismos, lo cual les permite un mayor nivel de contratación.

Los programas de reforma en México se han realizado siguiendo meticulosamente los puntos que se plasmaron en las agendas que el Banco Mundial le ha hecho llegar. México también presenta una fuerte influencia de los Estados Unidos de Norteamérica, prueba de ello fue la realización del Quinto Foro Global sobre Reinvención del Gobierno, Innovación y Calidad en el Gobierno en el siglo XXI, que estuvo basado en la Agenda Presidencial de Buen Gobierno de México (elaborada de acuerdo a los lineamientos del Banco Mundial plasmados en el documento: Mexico, a Comprehensive Development Agenda). Los encargados de la reforma fueron la Secretaría de la Contraloría y Desarrollo Administrativo (posteriormente, Secretaría de la Función Pública), la Secretaría de Hacienda y Crédito Público y, a partir del año 2000, la Oficina de la Presidencia para la Innovación Gubernamental. En estas entidades, la mayoría de los funcionarios de las instancias encargadas de la reforma se distinguían por una probada experiencia en el sector privado.

En Nicaragua, la estructura de lineamientos de la reforma respondió a presiones externas, específicamente de la Agenda Internacional para el Desarrollo de los Estados Unidos (AID), el Banco Mundial, el Fondo Monetario Internacional y el BID. En este país se percibe que estos organismos internacionales funcionan en un esquema de condicionalidad cruzada e interrelacionada. De hecho con el apoyo del AID el gobierno de Nicaragua realizó una conferencia titulada Reinventando el Gobierno. Aunque en Nicaragua se habló de una reforma del Estado, en realidad ésta se tradujo más bien en una reforma del Poder Ejecutivo, es decir, del aparato central del gobierno, y nunca de los poderes del Estado Nacional. 
A pesar de la decidida intención de reducir el número de ministerios del gobierno, se verificó la creación de secretarías de Estado como instituciones gubernamentales que adaptaron las funciones de los ministerios desaparecidos. Además, en el afán de compactar el tamaño del gobierno, también se produjo un ambiente de discrecionalidad en la contratación "no permanente" de recursos humanos calificados en las esferas altas del gobierno, lo que elevó el gasto corriente y el endeudamiento del país (Vicher, 2008: 296).

En el caso de Uruguay, se habla de que los parámetros que inspiraron la reforma fueron el Consenso de Washington y el ajuste estructural, que estuvieron fuertemente vinculados a la idea del Estado "mínimo" del Fondo Monetario Internacional. Por su parte, en Uruguay se afirma que los fundamentos de la reforma provienen de la tendencia general de aplicación de los nuevos principios de la gerencia pública. La evaluación de la gestión recayó en el Comité Ejecutivo para la Reforma del Estado que para desempeñar su tarea se valió de un grupo de consultores nacionales y extranjeros.

La NGP se ha aplicado en Uruguay, pero se ha observado que a pesar de la implementación de estas medidas, las empresas de mercado y las organizaciones del tercer sector siguen siendo dependientes del aparato estatal en la financiación, la elaboración de las políticas públicas y el desarrollo de las capacidades para cumplir sus cometidos; en mucho porque las organizaciones privadas tienen baja capacidad para reunir recursos y capacidades en función de intereses públicos (Vicher, 2008: 310). Además, se ha constatado que el traspaso de funciones públicas a organizaciones privadas puede traducirse en pérdida de eficacia y efectividad de las mismas, o bien en la falta de continuidad, que pueden llegar, incluso, al abandono del cumplimiento. En síntesis, se ha observado que las transferencias de funciones al mercado y al sector privado no han producido una reducción del gasto de la administración central.

El caso de Venezuela muestra que, aunque un gobierno de izquierda esté en el poder, ello no significa que las estrategias neogerenciales no se instrumenten. Por ejemplo, en el sistema de servicio civil se han desarrollado iniciativas sobre gestión del rendimiento, no obstante, se ha encontrado que es poco efectiva ya que no existen estándares de rendimiento aplicables a determinados tipos de empleados, además de que la evaluación depende de que se establezcan objetivos de desempeño individual y los supervisores no realizan oportunamente esta actividad. También se ha detectado que no existe una adecuada vinculación entre los criterios y mecanismos de promoción y la evaluación del rendimiento, así como entre el potencial de desarrollo y las competencias de los funcionarios.

En suma, las reformas neogerenciales analizadas en los países seleccionados, nos muestran que el fenómeno está presente y que el término NGP se utiliza, salvo algunas excepciones, de forma muy normal, no obstante que los resultados esperados no siempre coincidan con lo que esperaban los gobiernos cuando emprendieron dichas reformas. 


\section{5. ¿Contribuyeron las reformas neogerenciales a mejorar las capacidades del gobierno?}

El cambio en el paradigma de la gerencia pública en los países desarrollados que han impulsado dichas transformaciones, no ha sido uniforme, integral y homogéneo. Por el contrario, son diversos, en algunos casos radicales -Reino Unido, Australia y Nueva Zelanda-, en otros casos, limitados y conservadores -Francia y Portugal-, lo que permite sostener que el proceso de transformación de las reformas neogerenciales es simbólico y no necesariamente estructural.

Mientras en los países en desarrollo, en la década de 1980, cuando comienzan las reforma neogerenciales, la democracia ya ha avanzado extraordinariamente, haciendo con ello viable una forma más flexible de administración pública, en la cual un incremento de la autonomía de los gerentes públicos es acompañado con una mayor responsabilidad democrática (Bresser, 2004: 7). Lo que la reforma neogerencial plantea es que ahora el régimen político ha progresado lo suficiente como para ser consistente con una administración pública más eficiente, en la medida en que es más autónoma. Dicha reforma plantea que, tanto los políticos como los servidores públicos, efectivamente, requieren que sean responsables, pero que ahora existen nuevos y mejores mecanismos de responsabilidad.

Sin embargo las estrategias adoptadas para modificar los modelos tradicionales de implementación de las políticas públicas, muestran que las reformas de la NGP no han logrado superar las debilidades de la gestión pública, debido a su carácter disperso y parcial, así como por la insuficiente consideración de las problemáticas institucionales y la ausencia de esfuerzos para superar dichas limitaciones (Martínez, 2000: 11).

Las reformas neogerenciales deben aprender de las experiencias realizadas, tomando en cuenta tres consideraciones. En primer lugar, es erróneo avanzar en la modificación de los modelos de gerencia pública establecidos sin atender, a la vez, a los requerimientos de la construcción institucional, de las políticas sustantivas y de los procesos de movilización de los actores, tanto en las etapas de formulación como de ejecución. Las políticas no consisten simplemente en la enunciación de propósitos, definición de estrategias y sanción de los cuerpos legales, sino que constituyen un ciclo en el que la implementación determina el impacto sobre la realidad.

En segundo lugar, es preciso construir visiones más maduras acerca de las capacidades del Estado para alimentar las acciones de reforma neogerencial. En el pasado, fue usual la construcción de capacidades administrativas mediante la adquisición de insumos y equipamiento, de la construcción de infraestructura, de la capacitación del personal y de la aplicación de nuevos sistemas normativos. Aunque fueron útiles, los nuevos enfoques no deben confundir la superación de la carencia de medios con el incremento en las capacidades de gobernanza, para la gerencia pública y para la producción de impactos significativos. 
Por último, las intervenciones dirigidas a las reformas neogerenciales deben ser complejas y adecuadas a la naturaleza de las problemáticas que enfrentan y a las concepciones que gobiernan el diseño de las políticas. Los cambios puntuales y la introducción de nuevas modalidades de innovación en las tecnologías gerenciales, sólo son sustentables si responden a estrategias de cambio institucional y del entorno organizacional.

Las reformas a la administración pública necesitan determinar sus propias prioridades y guiarse por las necesidades de cada situación específica. Las reformas bajo la NGP son mucho más que las "tres E" (economía, eficiencia y eficacia), por lo que se sugiere incluir dos más: experimentación y eclecticismo. La búsqueda de soluciones a los problemas de gobierno en los países en desarrollo requiere de una visión más amplia que permita apegarse y adecuarse a las necesidades específicas y particulares de cada nación.

Las reformas neogerenciales han tenido diferentes resultados en los países de la OCDE (Ormond y Löffler, 1999: 3), por lo que se deben tomar en cuenta sus experiencias. Primero, no existen modelos previamente establecidos. El cambio debe enraizarse en la configuración específica de la historia, las tradiciones y estructuras constitucionales y legales, las fuerzas político-administrativas, las perspectivas económicas y sociales, así como la posición internacional de cada país. No existe una solución única para el sector público como un todo.

Segundo, la mayoría de los elementos de la NGP no son realmente nuevos en el sentido de que numerosos países han implementando en el pasado algunos de estos elementos, 0 con otras denominaciones. Lo que es "nuevo" en relación a la NGP es que el concepto global ha evolucionado a partir de un movimiento orientado a la práctica, que se inició en forma independiente y aislada en varios países.

Tercero, los países que han adoptado este enfoque de manera gradual, ahora confrontan un problema de estructuras duales en el sector público: islas de reforma con orientación hacia la NGP que coexisten con organizaciones públicas gerenciales en forma tradicional. Esta situación genera tensiones en el proceso de reformas, como resultado de estructuras de incentivos en conflicto y, en algunos casos, inclusive, llega a poner en tela de juicio lo que ha sido logrado hasta el momento.

Cuarto, existen también importantes desafíos concernientes a la gestión de gobierno, que no necesariamente están incluidos en el debate sobre la NGP. Algunos de los cuales pueden haber sido exacerbados por dicha reforma como son el mantenimiento de toma de decisiones efectivas del gobierno central, en un contexto de devolución y globalización; gerencia de la ética en el gobierno; educación y responsabilidad cívica en la sociedad; nuevas relaciones entre el ejecutivo y el sector privado; y las nuevas relaciones cambiantes entre ejecutivo y legislativo.

Por lo anterior, es indispensable recuperar la dimensión democrática de la NGP (Brugué, 1996: 45). Recuperar la dimensión democrática de la administración pública supone también 
aceptar que los conflictos, las luchas, los valores o los intereses que se entrecruzan en su interior no deben resolverse sólo a través de los mecanismos mercantiles, sino mediante los instrumentos políticos basados en la negociación y el consenso. La gestión de la administración pública debe orientarse a la configuración de un determinado modelo de sociedad, donde sus actuaciones sirvan para darle una forma determinada y, por lo tanto, incorporar una elección que no está exenta de conflictos. La administración pública configura un espacio donde surge y se resuelven conflictos, donde interactúan intereses contrapuestos, donde se prioriza entre valores en competencia y donde, en definitiva, se dan cita todos los elementos de un debate político.

En ese sentido, la gestión pública tiene que ser concebida entonces en su amplio sentido social, político y económico, además de ser entendida en los parámetros de la vida organizacional de las instituciones públicas. Lo que la administración pública hace o deja de hacer no sólo es producto de disposiciones jurídicas, sino también de un conjunto de adaptaciones constantes de las instituciones públicas a las condicionantes del entorno social, económico y político (Motta, 1991: 11). Por ello, a pesar de su relevancia, el análisis jurídico posee sus límites para entender lo que realmente sucederá en la gestión pública.

La ineficiencia y la debilidad institucional de las organizaciones públicas pueden tener su origen en la falta de actualización administrativa o la carencia de habilidades gerenciales. Tanto los análisis más simples de la gerencia pública como los diagnósticos para reformas administrativas revelan que ambos aspectos son determinantes para ofrecer los resultados que espera la sociedad (Motta, 1991: 9).

La problemática administrativa, y su relación con la infraestructura política, pueden ser mejor entendidas cuando se analizan las funciones desempeñadas por el Estado en la sociedad. Los gobiernos mediante leyes procuran determinar funciones, papeles y fuentes de legitimidad para la administración pública. A pesar de las concepciones legales y constitucionales, el éxito de la administración democrática depende tanto de la práctica política de esos valores como de las formas gerenciales que se utilizan en la administración de los asuntos públicos.

La ausencia de una práctica de administración democrática produce en la población incredulidad, desconfianza y falta de credibilidad respecto a la capacidad gubernamental. Por eso, paralelamente a las propuestas legales y a las reformas administrativas, es necesario recuperar constantemente la credibilidad de la población en la capacidad administrativa de los gobiernos para responder al interés público, además de reforzar las bases éticas y morales de la acción gubernamental mediante mayores mecanismos de legitimidad (Motta, 1991: 10).

Existen cuatro características que afectan la capacidad de gobierno (Alcántara, 1995: 30 31). La primera es el grado de calidad de la burocracia que garantice de manera suficiente asesoramiento y liderazgo en el proceso de formulación y puesta en marcha de las políticas 
públicas. La segunda, se refiere al compromiso de la burocracia con los objetivos del gobierno del momento en contraposición con sus intereses en tanto que organización e individuos. En tercer lugar, el entorno institucional en el proceso de gestación de políticas públicas por parte del gobierno puede influir en la gobernabilidad. Por último, se señala que el sistema de partidos puede asimismo influir en la gobernabilidad, especialmente en lo relativo a la habilidad para generar y poner en marcha mandatos claros de acción.

El modelo de la NGP se encuentra, entonces, en tela de juicio debido a que intenta representar un modelo económico universal de gobierno y organización que centra su atención en la eficiencia. Sin embargo, poco se ha hecho en lo que respecta a la evaluación de los efectos de la reformas del modelo de la NGP, ni en lo relacionado con la consecuencia de la importante meta de la eficiencia, ni en lo que se refiere a los efectos sobre otras metas, como la de la gobernaza política, lo cual se debe parcialmente al hecho de que no se trata de una tarea fácil (Christensen y Laegreid, 2001: 55).

Para estos autores existen pocas pruebas de que la aplicación de las estrategias de reforma basadas en el modelo de la NGP produzca realmente los resultados esperados. A menudo, los efectos y consecuencias del modelo de la NGP se dan por supuestos o se prometen, pero no se documentan. Dichos efectos son difíciles de cuantificar y muy controvertidos; se argumentan en contra de las relaciones entre las causas y los efectos; los indicadores de eficiencia y efectividad son elusivos; los efectos secundarios son difíciles de rastrear; y la metodología que se utiliza en la evaluación del modelo de la NGP es insuficiente (Christensen y Laegreid, 2001: 56).

\section{A manera de conclusiones}

El concepto de NGP es una estrategia de decisiones sobre políticas a seguir en los pasados veinte años, que ha redundado en un giro sustancial en el ejercicio de los gobiernos de Reino Unido, Nueva Zelanda, Australia, Escandinavia y América del Norte, es decir, especialmente en países anglosajones. La NGP intenta ser una fórmula utilizada regularmente por especialistas y profesionales para referirse a los temas, estilos y patrones distintivos de la gestión de la función pública moderna.

Las reformas gubernamentales en el mundo han tenido diversos enfoques, estrategias y resultados. Para analizar la influencia de la NGP en el mundo, se muestra que de cuatro modelos -Anglosajón, Europeo Continental, Europeo Septentrional y Países en Transición-, la NGP no es un modelo universal y aplicable a todos los países, sino que depende del desarrollo administrativo, de la fortaleza de las instituciones administrativas, las condiciones internas y externas de cada apartado gubernamental.

Las reformas neogerenciales, efectuadas en algunos de los países como Argentina, Chile, Bolivia, Colombia, Nicaragua, México, Uruguay y Venezuela, permiten confirmar que 
las estrategias de la NGP no fueron aplicadas de manera homogénea, como en los países anglosajones; muchas fueron "islas" de modernidad coexistendo con sistemas tradicionales de administración y, en general, mostraron más imitación de la NGP que innovación administrativa.

Resulta más evidente que el cambio en el paradigma de la gerencia pública, tanto en países desarrollados como de reciente industrialización, que han impulsado dichas transformaciones, no ha sido uniforme, integral y homogéneo, lo que permite sostener que el proceso de transformación de las reformas neogerenciales ha sido simbólico, como parte de la "moda administrativa" en boga, por lo que no fue, necesariamente, estructural.

Las enseñanzas que pueden aprender los países hispanoamericanos de sus reformas neogerenciales pueden ser muchas y variadas. Primero, es erróneo avanzar en la modificación de los modelos de gerencia pública establecidos sin atender los requerimientos de la construcción institucional, de las políticas sustantivas y de los procesos de movilización de los actores, tanto en las etapas de formulación como de ejecución.

Segundo, es preciso construir visiones más maduras acerca de las capacidades del Estado para alimentar las acciones de reforma neogerencial. En el pasado fue usual la construcción de capacidades administrativas mediante la adquisición de insumos y equipamiento, de la construcción de infraestructura, de la capacitación del personal y de la aplicación de nuevos sistemas normativos. Tercero, aunque fueron útiles, los nuevos enfoques no deben confundir la superación de la carencia de medios con el incremento en las capacidades de gobernanza, para la gerencia pública y para la producción de impactos significativos.

Por último, las intervenciones dirigidas a las reformas neogerenciales deben ser complejas y adecuadas a la naturaleza de las problemáticas que enfrentan y a las concepciones que gobiernan el diseño de las políticas. Los cambios organizacionales como la introducción de nuevas modalidades de innovación en las tecnologías gerenciales, sólo son sustentables si responden a estrategias de cambio institucional y del entorno organizacional.

Hace falta revisar las enseñanzas y aprendizaje de la aplicación de las reformas neogerenciales a la luz de los nuevos requerimientos de la gobernanza, la mayor participación de la ciudadanía en los asuntos de gobierno, en la transparencia y rendición de cuentas de los gobernantes, pero, sobre todo, tratar de responder en qué medida estas reformas fortalecieron las capacidades administrativas, pero debilitaron las capacidades políticas para alcanzar una plena democracia participativa. 


\section{Referencias Bibliográficas}

Alcántara Sáez, Manuel. 2004. Gobernabilidad, Crisis y Cambio. México: Fondo de Cultura Económica.

Arellano Gault, David, José Ramón Gil, Jesús Ramírez Macías y Ángeles Rojano. 2000. Nueva Gerencia Pública en Acción: Procesos de Modernización Presupuestal: un Análisis Inicial en Términos Organizativos (Nueva Zelanda, Reino Unido, Australia y México). Revista del CLAD. Reforma y Democracia 17: 7-44.

Aucoin, Meter. 1995. The New Public Management: Canada in Comparative Perspective. Montreal: IRPP. 1996. Reforma Administrativa en la Gestión Pública: Paradigmas, Principios, Paradojas y Péndulos. En Lecturas de Gestión Pública, compilado por Brugué, Quim y Joan Subirats, 491-515. Madrid: Ministerio de Administraciones Públicas-Instituto Nacional de Administración Pública.

Bardach, Eugene. 1998. Getting Agencies to Work Together: The Practice and Theory of Managerial Craftsmanship. Washington, D.C.: Brookings.

Barzelay, Michael. 1998. Atravesando la Burocracia. Una Nueva Perspectiva de la Administración Pública. México: Colegio Nacional de Ciencias Políticas y Administración Pública-Fondo de Cultura Económica. 2000. The New Public Management: Improving Research and Policy Dialogue. Berkeley: University of California Press.

2003. La Nueva Gestión Pública. Un Acercamiento a la Investigación y al Debate de las Politicas. México: Fondo de Cultura Económica.

Boston, Jonathan, John Martín, June Pallot y Pat Walsh. 1991. Reshaping the State: New Zeland's Bureaucratic Revolution. Auckland, New Zealand's: Oxford University Press.

Bozeman, Barry (comp.). 1999. La Gestión Pública. Su Situación Actual. México: Colegio Nacional de Ciencias Políticas y Administración Pública, Universidad Autónoma de Tlaxcala, Fondo de Cultura Económica.

Bresser-Pereira, Luiz Carlos et al. 2004. Política y Gestión Pública. Buenos Aires: Fondo de Cultura Económica, Centro Latinoamericano de Administración Pública para el Desarrollo.

Brugué, Quim. 1996. La Dimensión Democrática de la Nueva Gestión Pública. Gestión y Análisis de Políticas Públicas 5-6: 45-58.

Cabrero Mendoza, Enrique. 1997. Del Administrador al Gerente Público. México: Instituto Nacional de Administración Pública.

.2005. La Nueva Gerencia Pública y los Procesos de Reforma Gubernamental en Municipios de América Latina: ¿Avance o Retroceso para una Gobernanza Democrática? Ponencia presentada al $X$ Congreso Internacional del CLAD sobre la Reforma del Estado y de la Administración Pública, 18-21 de octubre, Santiago, Chile.

Campbell, Colin y John Halligan. 1992. Political Leadership in a Age of Constraint: The Australian Experience. Pittsburgh, P.A.: University of Pittsburgh Press. 
Campbell, Colin y Wilson, Graham. 1995. The End of Whitehall: Death of a Paradigm? Oxford: Blackwell.

Conde Martínez, Carlos. 2001. Los Procesos de Innovación y Cambio en la Gestión Pública de los Países de la OCDE. En La Nueva Gestión Pública, coordinado por Olías de Lima, Blanca, 369-395. Madrid: Prentice Hall.

Cristensen, Tom y Per Laegreid. 2001. La Nueva Administración Pública: el Equilibrio entre la Gobernanza Política y la Autonomía Administrativa. Gestión y Políticas Públicas X (1): 55-100.

Ferlie, Ewan, Andrew Pettigrew y Louise Fitzgerald. 1996. The New Public Management in Action. Oxford: Oxford University Press.

Guerrero, Omar. 1999. Del Estado Gerencial al Estado Cívico. México: Universidad Autónoma del Estado de México, Miguel Ángel Porrúa.

.2003a. Gerencia Pública en la Globalización. México: Universidad Autónoma del Estado de México, Miguel Ángel Porrúa.

2003b. Nueva Gerencia Pública: ¿Gobierno sin Política? Revista Venezolana de Gerencia 8 (23): 379-395.

Haywood, Stuart y Jeff Rodríguez. 1994. Un Nuevo Paradigma para la Gestión Pública. En El Rediseño del Estado. Una Perspectiva Internacional, compilado por Kliksberg, Bernardo. México, 189-207. México: Instituto Nacional de Administración Pública, Fondo de Cultura Económica.

Hood, Christopher. 1994. Explaining Economic Policy Reversals. Buckingham: Open University Press.

Hood, Christopher y Jackson, Michel. 1997. La Argumentación Administrativa. México: Colegio Nacional de Ciencias Políticas y Administración Pública, Universidad Autónoma de Coahuila, Fondo de Cultura Económica.

Kettl, Donald. 1995. Building Lasting Reform: Enduring Questions, Missing Answers. En Incide the Reinvention Machine: Appraising Governmental Reform, compilado por Donald Kettl y John Di lulio Jr., 9-83. Washington DC: Brookings.

Lane, Jan-Erick. 2000. New Public Management. London: Routledge.

Lynn, Lawrence. 1998. Public Management as Art, Science and Profession. Chatham, New Jersey: Chatham House.

2004. Reforma a la Gestión Pública: Tendencias y Perspectivas. En De la Administración Pública a la Gobernanza, compilado por Pardo, María del Carmen, 105-128. México: El Colegio de México.

Martínez Nogueira, Roberto. 2000. La Gestión Pública: del Diálogo Crítico a la Evaluación de la Diversidad. Política y Gestión 1: 171-196. 
Martínez Vilchis, José. 2007. Nueva Gerencia Pública. Un Análisis Comparativo de la Administración Pública en México. México: Universidad Autónoma del Estado de México, Facultad de Ciencias Políticas y Administración Pública, Miguel Ángel Porrúa.

Moore, Mark H. 1998. Gestión Estratégica y Creación de Valor en el Sector Público. Barcelona, Paidós.

Motta, Paulo Roberto. 1991. Innovación y Democratización de la Gestión Pública. Caracas: Centro Latinoamericano de Administración para el Desarrollo.

Ormond, Derry y Ele Löffler. 1999. Nueva Gerencia Pública: qué Tomar y qué Dejar. Revista del CLAD. Reforma y Democracia 13: 141-172.

Osborne, David y Ted Gaebler. 1994. Un Nuevo Modelo de Gobierno. Como Transforma el Espíritu Empresarial al Sector Público. México: Gernika.

Pollitt, Christopher y Geert Bouckaert. 2000. Public Management Reform. Oxford: Oxford University Press.

Richards, Sue. 1994. El Paradigma del Cliente en la Gestión Pública. Gestión y Análisis de Políticas Públicas 1: 5-16.

Sánchez González, José Juan. 2006. Gestión Pública y Governance. México: Instituto de Administración Pública del Estado de México.

Schick, Allen. 1996. The Spirit of Reform: Managing the New Zealand State Sector in a Time of Change. Wellington: State Services Commission and the Treasury.

Schwartz, Herman. 1994. Small States in Big Trouble. World Politics 46 (4): 527-555.

Tendler, Judith y Sara Freedheim. 1994. Trust in a Rent-Seeking World: Health and Government Transformed in Northeast Brazil. World Development 22 (12): 1771-1791.

Vicher García, Diana. 2008. La Transformación de la Administración Pública Hispanoaméricana: de la Reforma Administrativa a la Reforma Gerencial. Tesis (Doctorado en Ciencias Políticas y Sociales) Universidad Nacional Autónoma de México.

José Juan Sánchez González es Profesor a tiempo completo en la Universidad Autónoma del Estado de México. Licenciado en Ciencias Políticas y Administración Pública por la Facultad de Estudios Superiores "Acatlán"-UNAM. Maestro y Doctor en Administración Pública por la Universidad Nacional Autónoma de México (UNAM). Ha obtenido el Premio Instituto Nacional de Administración Pública (1997) y una Mención Honorífica del Premio Instituto de Administración Pública del Estado de México (2001). Es miembro del Instituto Nacional de Administración Pública y Miembro de Número del Colegio Nacional de Ciencias Políticas y Administración Pública. Pertenece al Sistema Nacional de Investigadores como Investigador Nacional nivel I. Ha publicado cinco libros sobre teoría de la administración pública y la administración pública mexicana y es autor de más de veinte artículos especializados en la disciplina y seis capítulos en libros colectivos. 
